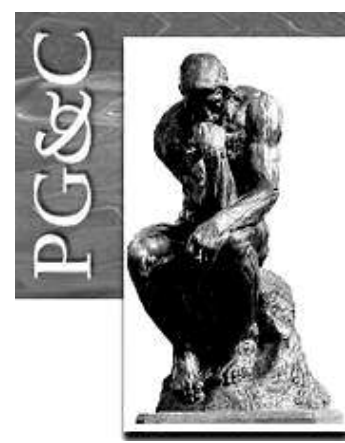

\title{
GESTÃO DO CONHECIMENTO NAS ORGANIZAÇÕES: FOCO NA QUALIDADE DA INFORMAÇÃO DISPONÍVEL EM OBSERVATÓRIOS
}

\author{
Cleunisse Aparecida Rauen De Luca Canto \\ Doutoranda em Engenharia e Gestão do Conhecimento pela Universidade \\ Federal de Santa Catarina, Brasil. Professora da Faculdade SENAI \\ Florianópolis, Brasil. \\ E-mail: cleocanto@gmail.com \\ Rogério Cid Bastos \\ Doutor em Engenharia de Produção pela Universidade Federal de Santa \\ Catarina, Brasil. Professor da Universidade Federal de Santa Catarina, \\ Brasil. \\ E-mail: rcbreaa@gmail.com
}

\section{Lia Caetano Bastos}

Doutora em Engenharia de Produção pela Universidade Federal de Santa Catarina, Brasil. Professora da Universidade Federal de Santa Catarina, Brasil.

E-mail: lia@ecv.ufsc.br

\begin{abstract}
Resumo
O século XXI vem provocando mudanças, já que saímos da sociedade industrial para adentrarmos na sociedade do conhecimento. Nesta nova era a informação é vasta, está em todos os lugares e é acessível a todos, mas quantidade não representa qualidade. Lidar com estas informações e gerar conhecimentos para as organizações úteis é o foco dos Observatórios. Assim, com o objetivo de identificar como é avaliada a qualidade da informação disponibilizada por Observatórios realizamos uma revisão da literatura, de forma sistemática, com base no check-list PRISMA e apresentaremos aqui sua versão revisada e ampliada. As bases de dados utilizadas foram Emerald, ERIC, Science Direct, Scopus e Web of Science, além do Google Scholar e ProQuest. As buscas aconteceram em duas etapas, inicialmente em setembro de 2017 com atualização em maio de 2018. Dos 20 artigos selecionados, oito foram alvo deste estudo, já que atendiam aos critérios de elegibilidade. Os resultados apontaram para a importância da qualidade das informações disponibilizadas pelos Observatórios analisados, já que estas interferem na tomada de decisão das organizações. Concluímos que o uso de metodologias, métodos ou ferramentas estruturadas para avaliar a qualidade da informação devem ser incorporadas nas estratégias dos Observatórios, já que estas direcionam as ações, delimitam gargalos e fornecem indicadores do que precisa ser melhorado.
\end{abstract}

Keywords: Qualidade da Informação. Observatórios. Avaliação. Organização. Gestão do Conhecimento.

KNOWLEDGE MANAGEMENT IN ORGANIZATIONS: FOCUS ON QUALITY OF INFORMATION AVAILABLE IN OBSERVATORIES

\begin{abstract}
The 21st century has brought about change, as we left industrial society to enter the knowledge society. In this new age information is vast, everywhere, and accessible to all, but quantity is not quality. Dealing with this information and generating knowledge for useful organizations is the focus of the Observatories. Thus, in order to identify how the quality of information provided by Observatories is
\end{abstract}

Perspectivas em Gestão \& Conhecimento, João Pessoa, v. 9, n. 3, p. 64-80, set./dez. 2019. DOI: http://dx.doi.org/10.21714/2236-417X2019v9n3p64

http://periodicos.ufpb.br/ojs2/index.php/pgc. ISSN: 2236-417X. Publicação sob Licença (cc) EY-NC-ND 
evaluated, we systematically review the literature based on the PRISMA checklist and present its revised and expanded version. The databases used were Emerald, ERIC, Science Direct, Scopus and Web of Science, as well as Google Scholar and ProQuest. The searches took place in two stages, initially in September 2017 and updated in May 2018. Of the 20 articles selected, eight were the subject of this study, as they met the eligibility criteria. The results pointed to the importance of the quality of the information provided by the Observatories analyzed, as they interfere in the decision making of organizations. We conclude that the use of structured methodologies, methods or tools to assess the quality of information should be incorporated into the Observatories' strategies, as they direct actions, delimit bottlenecks and provide indicators of what needs to be improved.

Keywords: Quality information. Observatory. Evaluation. Organizations. Knowledge management.

\section{INTRODUÇÃO}

O interesse em identificar como a literatura trata a qualidade da informação disponibilizada por observatórios foi alavancada a partir de estudos sobre o modelo de negócio do 'Observatório da Indústria Catarinense', da Federação das Indústrias do Estado de Santa Catarina (FIESC). Este observatório, conforme claramente definido em seu site, "monitora os principais fatores que afetam a competitividade industrial no Estado de Santa Catarina, analisando o desempenho econômico e as tendências tecnológicas dos setores estratégicos, fornecendo informações para a tomada de decisões, tanto na esfera estadual quando regional" ${ }^{1}$. Empresários, gestores públicos e universidades utilizam esta informação como suporte ao planejamento e ao desenvolvimento estratégico da indústria no Estado de Santa Catarina.

O que se observa neste contexto é que a informação é um subsídio de extrema relevância para a tomada de decisão. Informação esta que precisa ser clara, objetiva, relevante e, principalmente, de qualidade, pois impacta na gestão de conhecimentos da organização.

De acordo com o Dicionário Aurélio, "a qualidade é uma das categorias fundamentais do pensamento: maneira de ser que se afirma ou se nega de uma coisa" (FERREIRA, 1975, p. 1165). É difícil, porém, dizer qual informação que tem qualidade, ou ainda, quais são os parâmetros a serem observados no processo de seleção de uma informação de boa qualidade, uma das maiores preocupações das organizações na atualidade, na chamada sociedade do conhecimento.

Para Oleto (2006), pensar sobre a qualidade da informação e como esta impacta nas decisões das organizações é, por si só, um requisito imprescindível neste mundo globalizado, onde a informação é vasta, está em todos os lugares, é disponibilizada de diferentes formas e está acessível a todos, e em grande quantidade.

Não temos dúvidas que, segundo apontaram Gualazzi, Santos e Campos (2013), avaliar a qualidade da informação é um grande desafio, mas é uma possibilidade para se identificar pontos fracos por meio de seus atributos de qualidade, quantificando aquilo que não é subjetivo, a satisfação. Conforme enfatizaram Li e Lin (2006), é a qualidade da informação que contribui positivamente para a satisfação dos clientes e, consequentemente, para a qualidade de parcerias de negócio ao longo da cadeia de suprimentos.

Para entender como avaliar esta qualidade, Canto, Bastos e Bastos (2019) realizaram uma revisão sistemática da literatura sobre o tema que resultou no artigo intitulado 'Avaliação da Qualidade da Informação disponibilizada por Observatórios: revisão sistemática da

\footnotetext{
${ }^{1}$ Disponível em: http://fiesc.com.br/pt-br/observatorio-da-industria-catarinense. Acesso em julho de 2019.
}

Perspectivas em Gestão \& Conhecimento, João Pessoa, v. 9, n. 3, p. 64-80, set./dez. 2019. 
literatura'. Este artigo foi apresentado no '30ㅡ ENANGRAD: Gestão da Aprendizagem no Contexto das Transformações' e será a base deste estudo. Para tanto, esta versão revisada e ampliada aprofundará os conceitos de informação, qualidade da informação e gestão do conhecimento, bem como apresentará um método detalhadamente descrito, resultados reestruturados e dispostos em formato textual, mas sem perder a base do artigo original.

O artigo objetiva, então, revisar e detalhar os estudos obtidos da revisão sistemática da literatura proposta pelos autores para subsidiar a gestão do conhecimento nas organizações. Ele foi construído em cinco seções, na seção dois (2) apresentamos os conceitos basilares relacionados ao tema; na seção três (3) os procedimentos metodológicos; na seção quatro (4) a análise e a discussão dos resultados; e na seção cinco (5) as considerações finais. Por fim, na última seção, relacionamos as bibliografias utilizadas para esta análise.

\section{REVISÃO DA LITERATURA}

Dado, informação e conhecimento fazem parte do processo de transmissão de conhecimento. Números, fatos ou símbolos, quando sozinhos em sua forma bruta, nada mais são do que dados, pois não têm nenhuma estrutura inerente ou relacionamento entre eles mesmos. Ao estruturarmos o dado em algo que possamos fazer conclusões, com contexto ou significado, se transforma em informação. O conhecimento, no entanto, representa um nível mais alto de abstração e está relacionado ao processo cognitivo, resultando da inferência feita após a análise dos fatos relatados (HEY, 2006). No quadro 1 podemos visualizar o significado de dado, informação e conhecimento na perspectiva de Davenport e Prusak (1998).

Quadro 1 - Significado de dado, informação e conhecimento

\begin{tabular}{|c|c|c|}
\hline DADO & INFORMAÇÃO & CONHECIMENTO \\
\hline $\begin{array}{l}\text { Simples observações sobre o } \\
\text { estado do mundo. }\end{array}$ & $\begin{array}{c}\text { Dados dotados de relevância e } \\
\text { propósito. }\end{array}$ & $\begin{array}{l}\text { Informação valiosa da mente } \\
\text { humana. Inclui reflexão, síntese e } \\
\text { contexto. }\end{array}$ \\
\hline É facilmente estruturado. & Requer unidade de análise; & De estruturação difícil. \\
\hline É facilmente obtido por máquinas. & $\begin{array}{l}\text { Exige consenso em relação ao } \\
\text { significado. }\end{array}$ & De captura difícil em máquinas. \\
\hline Frequentemente quantificado. & \multirow{2}{*}{$\begin{array}{c}\text { Exige, necessariamente, a mediação } \\
\text { humana. }\end{array}$} & Frequentemente tácito. \\
\hline Facilmente transferível & & De transferência difícil \\
\hline
\end{tabular}

Fonte: Devenport e Prusak (1998)

Nonaka e Takeuchi, Davenport e Prusak, Peter Drucker e Stewart são, segundo Barbosa (2008), os precursores quando se trata de informação e de conhecimento. Segundo estes autores, a informação e o conhecimento se transformam, cada vez mais, em importantes fatores das mudanças econômicas e sociais. São temas que têm merecido a atenção de gestores, profissionais e pesquisadores devido a sua crescente importância para as organizações contemporâneas.

O conhecimento para Drucker (1969, p. 264) "[...] é o principal investimento e o principal produto da economia avançada, bem como o meio de vida do maior grupo da população". Assim, entender o significado da palavra conhecimento, diferenciando de dado e informação, é importante ao estudarmos a gestão do conhecimento.

Perspectivas em Gestão \& Conhecimento, João Pessoa, v. 9, n. 3, p. 64-80, set./dez. 2019. 
O que se observa na literatura, no entanto, é que o conceito de gestão do conhecimento está longe de ser um consenso, conforme reportado por Santos, Tecchio e Fialho (2014). Para os autores, as diferentes definições caracterizam-se como uma maneira pelo qual as organizações criam, armazenam, disseminam e usam o conhecimento, conforme podemos visualizar no Quadro 2:

Quadro 2 - Definições para o termo gestão do conhecimento

\begin{tabular}{|l|l|}
\hline AUTOR & CONCEITO \\
\hline $\begin{array}{l}\text { Nonaka e Takeuchi } \\
\text { (1997) }\end{array}$ & $\begin{array}{l}\text { A capacidade de uma organização de criar novo conhecimento, disseminá-lo } \\
\text { na organização e incorporá-lo em seus produtos, serviços e sistemas. }\end{array}$ \\
\hline $\begin{array}{l}\text { Davenport et al. } \\
\text { (1998) }\end{array}$ & $\begin{array}{l}\text { A gestão do conhecimento é um processo de coleta, distribuição e utilização } \\
\text { eficiente dos recursos de conhecimento em toda a organização. }\end{array}$ \\
\hline Bhatt (2001) & $\begin{array}{l}\text { Um processo de criação, validação, apresentação, distribuição e aplicação que } \\
\text { permitem a uma organização aprender, refletir, desaprender e reaprender, } \\
\text { sendo estas cinco fases consideradas essenciais para construção, manutenção } \\
\text { e reabastecimento das competências principais. }\end{array}$ \\
\hline $\begin{array}{l}\text { Na Ubon e Kimble } \\
\text { (2002) }\end{array}$ & $\begin{array}{l}\text { Gestão do conhecimento refere-se ao gerenciamento de processos que } \\
\text { governam a criação, disseminação e utilização do conhecimento por meio da } \\
\text { fusão de tecnologias, estruturas organizacionais e pessoas para criar um } \\
\text { aprendizado mais efetivo, resolver problemas e tomar decisão em uma } \\
\text { organização. }\end{array}$ \\
\hline Lopez et al. (2004) & $\begin{array}{l}\text { Gestão do Conhecimento é um processo que facilita a partilha de } \\
\text { conhecimentos e estabelece aprendizagem como um processo contínuo } \\
\text { dentro de uma organização. }\end{array}$ \\
\hline
\end{tabular}

Fonte: Santos, Tecchio e Fialho (2014)

A Gestão do Conhecimento é, portanto, uma combinação complexa de pessoas, processos e tecnologias. O conhecimento provém das pessoas e os aspectos humanos neste processo estão representados na dimensão tácita do conhecimento, na imaginação e na criatividade, na construção do sentido, na capacidade de interpretação múltipla, no caráter construtivista da criação e na renovação do conhecimento (CARVALHO, 2006). Os processos estão embutidos em um conjunto de possibilidades, desde o compartilhamento das informações nas organizações até a criação de novos conhecimentos, proporcionando vantagens competitivas e permitindo a disseminação a partir do uso de diferentes tecnologias (BACK, 2016). Refere-se, ainda, a utilização do conhecimento na resolução dos problemas organizacionais e como suporte a tomada de decisão, beneficiando a organização para o uso eficiente, tanto do conhecimento individual quanto do coletivo, com isto atingindo metas de maneira mais efetiva (CANTO et al., 2018).

Inserido neste cenário, os Observatórios configuram-se como espaços úteis para a tomada de decisão pelas organizações. Um Observatório, de acordo com Trzeciak (2009, p. 31), "é um mecanismo que fornece informações estratégicas para auxiliar na identificação de ameaças, oportunidades e tendências, além de oferecer subsídios à tomada de decisão de um determinado setor, seja em nível regional, nacional ou internacional".

Segundo Alves e Kern (2007 apud AMIN, 2010), um Observatório é um fenômeno tecnológico, pois suas ações acontecem de forma planejada, projetada e são dirigidas para cumprir algum tipo de finalidade. Neste contexto, a preocupação com a qualidade da informação e do conhecimento disponibilizado é fator preponderante. Conforme ressaltou Calazans (2008, p. 4), a "qualidade de informação é um dos alicerces para a sobrevivência e 
maior competitividade das organizações". Segundo o autor, avaliar, medir e aprimorar a qualidade da informação, permitindo uso e reuso pelas organizações, com maior eficiência e eficácia, tem sido o tema de diversos estudo, com as mais diferentes abordagens.

Nesta linha, o Observatório, "dispositivo de observação criado por um organismo para acompanhar a evolução de um fenômeno, de um domínio ou de um tema estratégico, no tempo e no espaço" (FIEP, 2004), é uma fonte poderosa para auxiliar na tomada de decisão nas organizações. Conforme ressaltaram Madnick et al. (2009), as organizações tem investido cada vez mais em tecnologias que permitam coletar, armazenar e processar grandes quantidades de dados, além de traduzir estes dados em ideias significativas que possam ser utilizadas para melhorar processos, tomar decisões inteligentes e criar vantagens estratégicas, algo que nem sempre é possível.

Assim, tratar de quantificar o valor da informação por meio de avaliação de seus atributos de qualidade, buscando indicadores que aponte para a efetividade das informações disponibilizadas, agrega valor e resulta na qualidade da informação prestada, conforme apontado nos estudos advindos das referências desta pesquisa.

\section{MÉTODO}

Esta é uma pesquisa de caráter exploratório que se utilizou de um estudo fundamentado por meio de uma revisão sistemática da literatura (RSL) para responder a seguinte questão de pesquisa: "Como é avaliada a qualidade da informação disponibilizada por observatórios?".

Uma revisão sistemática da literatura utiliza fonte de dados da literatura científica e conta, também, com procedimentos sistematizados de coleta e análise de dados, de forma que a pesquisa seja transparente e replicável (STEIL; PENHA; BONILLA, 2016). Nesta lógica, o estudo base deste artigo está fundamentado na RSL de Canto, Bastos e Bastos (2019) que seguiu o check-list Preferred Reporting Items for Systematic Reviews na Meta-Analyses (PRISMA), proposto por Moher et al. (2015), para a definição das etapas do estudo. Dentre os critérios adotados estão:

\section{a) Seleção}

Para a seleção dos dados partimos de diferentes bases de dados que tipicamente publicam pesquisas na área, sem critérios prévios, e obtivemos retorno nas bases Eric, Emerald, Scopus, Science Direct e Web of Science, além do Google Scholar e ProQuest.

As buscas foram realizadas durante o mês de setembro de 2017, com revisão em maio de 2018, a partir dos termos "Information Quality" AND "Observator*", por tópico, título, resumo e palavras-chave.

Os registros obtidos de cada fonte de dados foram exportados para o Thomson Reuters Endnote X7 (Clarivate Analytics, Filadélfia, PA, EUA), primeiramente em grupos separados e na sequência em um único grupo para a eliminação dos duplicados.

Selecionamos os artigos utilizando uma abordagem de três fases. Na primeira lemos títulos e resumos de forma independente, separando os selecionados em um novo grupo do Endnote $X 7$. Na segunda procedemos com a leitura dos resumos dos artigos selecionados na primeira fase e, novamente criamos um grupo independente para separar a amostra. $\mathrm{Na}$ terceira definimos os critérios de elegibilidade para leitura do artigo completo. Criamos dois novos grupos, um para os artigos excluídos e outro para a amostra final, os incluídos.

Perspectivas em Gestão \& Conhecimento, João Pessoa, v. 9, n. 3, p. 64-80, set./dez. 2019. 


\section{b) Critérios de elegibilidade}

Para os 20 artigos selecionados na amostra utilizamos os seguintes critérios para a inclusão: Observatórios como participantes; exposição baseada na qualidade da informação, com análises qualitativas, quantitativas ou ambas; sem padrão de comparação; e desfecho apontando a qualidade da informação disponibilizada. Não aplicamos restrições à linguagem ou tempo de publicação.

Os artigos excluídos foram catalogados com os seguintes critérios: (1) não reportavam observatório como fonte das informações; (2) não reportavam qualidade da informação como foco principal; (3) eram resumos, revisões, papers, livros ou abstract de conferências; e (4) não foram encontrados nas bases de dados ou não tiveram retorno do autor. A tabela 1 apresenta os estudos que foram excluídos e a razão para exclusão, seguindo a numeração acima.

Tabela 1 - Artigos excluídos e razão para exclusão

\begin{tabular}{clc}
\hline No & Autor & Razão para exclusão \\
\hline 1. & ALEXANDER, 2011 & 2 \\
2. & BOOTH, 2013 & 2 \\
3. & BOTHA, 2014 & 3 \\
4. & CLAUSEN, 1996 & 1 \\
5. & HAIQUN, 2013 & 1 \\
6. & METTLER, 2008 & 1 \\
7. & MANOUSELIS, 2006 & 1 \\
8. & MOHAMED, 2010 & 1 \\
9. & RADZIWILL, 2006 & 3 \\
10. & VAN CANEGHEM, 2012 & 1 \\
11. & VAN MOSSEVELD, 2016 & 3 \\
12. & VRÎNCIANU, 2009 & 2 \\
13. & YING, 2012 & 4 \\
14. & ZANG, 2006 & 2 \\
\hline
\end{tabular}

Fonte: Os autores

\section{c) Risco de Viés de cada estudo}

Avaliamos o risco de viés da metodologia dos estudos selecionados por meio do Best Practices for Survey Research Reports: A Synopsis for Authors and Reviewers da American Association for Public Opinion Research (AAPOR) (DRAUGALIS, 2008), que está estruturado com 10 questões norteadoras: (i) houve uma questão de pesquisa claramente definida?; (ii) as amostras representam bem a população a ser estudada?; (iii) os autores usam designs que equilibram custos com erros?; (iv) descreveram o instrumento de pesquisa?; (v) o instrumento foi pré-testado?; (vi) foram descritas medidas de controle de qualidade?; (vii) a taxa de resposta foi suficiente para permitir a generalização dos resultados para a população-alvo?; (viii) tem técnicas estatísticas e analíticas apropriadas aos dados coletados?; (ix) há evidência de tratamento de seres humanos?; e $(x)$ os autores foram transparentes para garantir a avaliação e replicação? Essas perguntas serviram como guia na identificação das características de qualidade da pesquisa com respostas padronizadas como 'Sim' (Yes), 'Não' (No), 'Pouco Claro' (Unclear) e 'Não Aplicável' (Not Applicable). Uma análise das 10 perguntas de suporte e a correlação de viés é fornecido no item 4.3.

\section{d) Medidas sumárias}

Perspectivas em Gestão \& Conhecimento, João Pessoa, v. 9, n. 3, p. 64-80, set./dez. 2019. 
Analisamos cada artigo para identificar as medidas comuns entre eles, avaliando as correlações existentes e suas similaridades, ou não, em todas as amostras.

\section{RESULTADOS}

Para a seleção dos estudos realizamos as buscas em duas fases, sendo a primeira em setembro/2017 e a segunda em maio de 2018. Adotamos este critério porque consideramos a amostra pequena e porque acabamos prorrogando a conclusão das análises.

Nas buscas realizadas na primeira fase, em setembro/2017, utilizamos 10 diferentes bases de dados e obtivemos retorno nas bases Eric, Scopus, Science Direct e Web of Science, com 39 referências. Prosseguimos com as buscas na ProQuest, onde conseguimos 9 referências, e no Google Scholar, que utilizamos como linha de corte a seleção dos 50 primeiros registros. Assim, para a amostra de 98 registros $(39+9+50)$ excluímos os duplicados, analisamos os títulos e os resumos, ficando com 20 artigos para a leitura de texto completo. Aplicando os critérios de inclusão, 13 deles foram excluídos.

Em maio de 2018 a busca foi atualizada para todas as bases de dados e incluímos a base Emerald, pois retornou 11 estudos. Google Scholar e ProQuest não passaram por atualização. Nesta segunda fase permanecemos com um total de 35 novos registros que foram exportados para o Endnote $X 7$. Comparamos as amostras e excluímos os duplicados. Observamos que 23 deles não estavam contemplados na primeira fase e se configuravam como potenciais amostras. Para estes fizemos uma análise dos títulos, na sequência do resumo e, finalmente, a leitura do artigo completo. Após aplicação dos critérios de elegibilidade só um (1) deles permaneceu.

Assim, na primeira fase selecionamos sete (7) estudos e na segunda um (1), totalizando oito (8) artigos que foram detalhadamente descritos nesta pesquisa. 0 processo completo de identificação, triagem, elegibilidade e inclusão foi descrito por Canto; Bastos; Bastos (2019) no estudo base e em formato de figura, que detalhamos aqui.

Dentre as medidas sumárias avaliadas, a única medida comum entre quatro dos oito estudos foi o Cronbach Alpha que foi superior a 0,70 e, consequentemente, significativo. As demais medidas são especificas de cada estudo e não passíveis de comparação. Mesmo os indicadores similares entre os estudos não possuem critérios idênticos de análise.

\subsection{Características do estudo}

Os oito estudos selecionados foram publicados entre os anos de 2006 e 2017. Seis estudos reportaram a pesquisa diretamente a um 'observatório' (MOHAMED, 2010; CARDUCCI et al., 2011; VAN DER LINDEN; LEEMPUT, 2015; PASCHALOUDIS; TSOURELA, 2015; BIANCHINI et al., 2011; GARCIA-PEREZ-DE-LEMA; MADRID-GUIJARRO; MARTIN, 2017; SHAH, 2015; PÉREZ; NASSIF, 2017) e dois estudos como 'escritório/associação' (SARAPAIVANICH; KOTEY, 2006; SHAH; SHAMAIL; AHMAD AKHTAR, 2015). Pela consistência das informações apresentadas durante leitura precisa dos dois artigos optou-se por mantê-los na amostra, e o critério para a inclusão foi a definição de Observatório proposta por Trzeciak (2009) na revisão deste estudo. Os resultados podem ser visualizados no tópico a seguir. 


\subsection{Resultado dos estudos individuais}

Apresentaremos uma síntese dos principais resultados obtidos na revisão sistemática da literatura. No artigo original os autores disponibilizaram uma tabela com as características descritivas dos artigos incluídos, mas nesta versão revisada apresentaremos em formato descritivo, considerando a difícil visualização dos dados quando alteramos a tabela para o padrão portrait.

Com o objetivo de analisar a forma como os dados sobre a quantidade de resíduos sólidos (MSW) é registrado, processado e relatado pelo Ronchi Decree Instituted the National Waste Observatory (Osservatorio Nazionale sui Rifiuti, ONR) para verificar a qualidade do sistema de gestão de resíduos italiano, Bianchini et al. (2011) realizaram o estudo intitulado Material and energy recovery in integrated waste management system - An Italian case study on the quality of MSW data. Os dados foram coletados a partir da análise quantitativa do Relatório anual publicado pelo ONR, questionários e declarações do Modello Unico di Dichiarazione Ambientale (MUD). Os instrumentos utilizados para a coleta de dados foram: identificação do tipo de organização, análise do ciclo da coleta, quantidade, tipo e fonte de MSW usado para projetar e planejar o sistema de gerenciamento de resíduos e para prever tendências futuras. A amostra contou com 139.189.668 toneladas de MSW e SW (special waste) produzidos no ano de 2005 em Bergamo, Bolonha, Brescia, Milão, Pádua e Turim. De acordo com os resultados obtidos, só Brescia alcançou a meta de $35 \%$ de nível de separação (source separation level - SSL); a quantidade de resíduos sólidos aumentou anualmente e elevou a coleção separada (SC); e a produção de resíduos sólidos não se configurou como um valor constante. Segundo os autores, o artigo focou em uma análise qualitativa e quantitativa para definição do nível de separação e influência de resíduos especiais incorporados aos Resíduos Sólidos Municipal (MSW). Uma investigação foi necessária para identificar parâmetros para o nível de separação geral (SSL). Concluíram que a qualidade das informações que definiram esses parâmetros é de fundamental importância para a projeção e a verificação do plano de manejo e identificação de possíveis ações de melhoria, não apenas para uma medida de desempenho do sistema de gerenciamento de resíduos. Ressaltaram que informações claras sobre quais tipos de coleta de lixo poderiam ser chamadas de coleção e como calcular o nível de separação são indispensáveis no contexto nacional e europeu.

No estudo Mass media health information: quantitative and qualitative analysis of daily press coverage and its relation with public perceptions, Carducci et al. (2011) objetivaram descrever os métodos seguidos pela Pisa University OCS (Osservatorio dela Comunicazione Sanitaria, ou Health Comunication Observatory) para coletar, armazenar e analisar artigos relacionados a saúde e seus conteúdos de banco de dados. Os autores analisaram 24.434 artigos, sendo 4.436 (18\%) relacionados à saúde, que foram publicados online nos jornais $L a$ Stampa, Il Corriere della Sera e La Republica, e que forneciam dados epidemiológicos sobre doenças transmitidas por alimentos. Os questionários foram respondidos por 492 pessoas nas unidades locais de saúde de Lucca e Pisa. Os dados estavam em um banco de dados de texto (DBT) e foram coletados e armazenados de 1999 a 2008. A qualidade foi medida a partir da correção-integridade (acurácia), confiabilidade, compreensão, utilidade, equilíbrio e independência. De acordo com os resultados, 32\% dos artigos estavam com erros ou incompletos; $19 \%$ estavam sem fonte de informação; $43 \%$ não ajudavam na tomada de decisão; $95 \%$ eram difíceis de ler; $37 \%$ possuíam palavras alarmantes; $16 \%$ eram tendenciosos; $70 \%$ das pessoas mudaram seus hábitos alimentares pela mídia; e o percentual de respostas corretas foi muito baixo. Os autores verificaram que grande parcela da população pesquisada disse ter mudado seus hábitos alimentares, pelo menos temporariamente, como consequência da informação da mídia. Concluíram que pesquisas populacionais cuidadosamente 
segmentadas, quando combinadas com monitoramento contínuo de mensagens de saúde em jornais e revistas, bem como com uma análise qualitativa e quantitativa da qualidade das informações disponibilizadas pode ser útil para estudar o impacto dos meios de comunicação sobre as percepções públicas, as atitudes e o conhecimento, talvez alterando efeitos e impedindo, ou corrigindo, erros e falsas declarações.

Garcia-Perez-de-Lema, Madrid-Guijarro e Martin (2017) analisaram o impacto das relações universitárias com as pequenas e médias empresas (PME), focando em inovação e desempenho, com base em dados recolhidos de um questionário administrado a uma amostra estratificada de 600 PME espanholas, no artigo intitulado Influence of university-firm governance on SMEs innovation and performance levels. Os dados foram colhidos de abril a setembro de 2011 por questionário ao CEO da empresa, sendo a população segmentada pela atividade industrial. Pré-teste para validação do questionário, além de entrevistas, testes t e qui-quadrado, análise de viés e uma triangulação metodológica foram realizadas. Para medir o controle da qualidade da informação tanto o viés de não-resposta quanto o viés de variância foram analisados. De acordo com os resultados: (a) empresas que empregam formas contratuais de governança apresentam maiores níveis de inovação; b) formas contratuais de governação são reforçadas por fatores relacionais com maior efeito de fortalecimento empresas mais jovens; e (c) o desempenho das PME é afetado pelo nível de inovação. O viés de variância do método (16\%) não foi significativo, também não houve diferença significativa entre os dois grupos. Os autores ressaltaram que orientação para inovação e atividades inovadoras são necessárias para as PME se tornarem, ou permanecerem, competitivas. Concluíram que a mobilização de canais de transferência feitas pelo SME Economic Observatory, um centro de investigação para as PME financiado pelo Instituto de Fomento da Região de Múrcia, por meio de formas complementares de governança, ajuda a construir uma ponte entre as relações pessoais e codificam o conhecimento, melhorando a inovação, a qualidade da informação disponibilizada e o desempenho de PME.

No estudo Using ES-QUAL to Measure Internet Service Quality of eBanking Web Sites in Greece, Paschaloudis e Tsourela (2015) objetivaram medir a qualidade dos sites que fornecem serviços eletrônicos no setor bancário da Grécia. Para tanto, investigaram a qualidade do serviço de websites de usuários cadastrados em sistemas de e-banking na Grécia a partir do Observatory for the Greek Information Society. De 800 questionários estruturados, os autores obtiveram 487 respostas válidas. Utilizaram as escalas ES-QUAL e E-RecS-QUAL para explorar as dimensões que influenciavam a percepção geral de qualidade do e-service, conforme escalas propostas por Parasuraman et al. (2005): eficiência, disponibilidade do sistema, cumprimento, privacidade, responsividade, compensação, contato e para percepções gerais da qualidade do e-service. Os resultados indicaram que o maior coeficiente de correlação esteve entre as percepções de qualidade de responsividade e de serviço eletrônico geral, sendo a Disponibilidade do sistema e Compensação as mais fracas. Em relação à importância relativa das dimensões das duas escalas, o "contato" foi evidenciado como o de melhor classificação e a "responsividade" como o segundo mais significativo. Quatro dimensões do ES-QUAL e 3 do ERecS-QUAL apresentaram correlações. Os resultados sugeriram haver uma correlação forte e positiva entre eles e a percepção geral dos sites bancários. De acordo com os autores, essa pesquisa pode ajudar a identificar os pontos fortes e fracos dos sites e sugerir ideias para melhorias na qualidade da informação disponibilizada. Concluíram que o estilo de vida e a cultura dos países afetam significativamente a percepção dos serviços online, e que o alto nível de qualidade do serviço eletrônico da Web é meta que todo banco deve tentar alcançar. Ressaltaram que a sociedade grega apresentou comprovada imaturidade quanto a qualidade.

Com o objetivo de identificar os fatores que influenciam na avaliação dos observatórios sociais no Brasil sob a perspectiva da Gestão de Informação, Pérez e Nassif

Perspectivas em Gestão \& Conhecimento, João Pessoa, v. 9, n. 3, p. 64-80, set./dez. 2019. 
(2017) realizaram uma análise em seis observatórios sociais brasileiros (Observatório Social do Brasil, Observatório de Políticas Públicas para a Agricultura, Observatório de Gestão da Informação, Observatório das Metrópoles, Observatório Itaú Cultural e Observatório da Diversidade Cultural) no artigo intitulado Fatores de influência na avaliação dos Observatórios Sociais do Brasil sob a perspectiva da gestão de informação. A coleta de dados foi realizada com 46 usuários a partir do nível de visibilidade dos sites onde estavam hospedados, tipo de usuário-alvo e tipo de produto e serviço fornecido. A pesquisa foi qualitativa e descritiva, caracterizando-se como um estudo de caso e contou com duas etapas: 1a) observação direta, constituída pela identificação das características gerais dos observatórios escolhidos (lugar, ano de criação, objetivos, serviços e produtos de informação fornecidos); 2a) aplicação de questionários (adaptação da CyberGuide) a usuários internos e externos para determinar os pontos em comuns e divergentes em cada caso. A pesquisa concluiu com a triangulação metodológica das duas etapas e a determinação dos fatores mais significativos para avaliar os observatórios sociais brasileiros. A qualidade da informação (QI), a atualidade da informação (AI) e os fornecedores da informação (FI) constituíram-se nos três parâmetros de maior influência na avaliação desses sistemas de monitoramento informacional. De acordo com os resultados, os observatórios são espaços de informação, intercâmbio e colaboração; QI e Al foram mais escolhidos $(35,2 \%)$ e são mais significativos (70\%); FI recebeu $14,4 \%$ dos votos; e a triangulação apontou a necessidade de melhoria na vigilância e no monitoramento. De acordo com os autores, esses fatores contribuem para a avaliação efetiva e correta dos observatórios sociais brasileiros, visando apoiar a geração de conhecimento e o desenvolvimento da inteligência social nos sistemas de vigilância informacional. Concluíram que as informações sobre os fatores de maior influência na avaliação dos OS (etapa 1), os de maior relevância (etapa 2) e a triangulação permitiram identificar que QI, Al e FI tem maior influência para os brasileiros.

A amostra de Sarapaivanich e Kotey (2006), no estudo The effect of financial information quality on ability to access external funds and performance of SMEs in Thailand, objetivou examinar o impacto da qualidade da informação financeira (QIF) na percepção de proprietários-gerentes para acessar capital e o efeito de duas variáveis na performance das pequenas e médias empresas (PME) na Tailândia. As PME estavam localizadas em Bangkok e nas províncias de Khon Kan e Chiang Mai, totalizando 407 PMEs, sendo que 220 delas foram entrevistadas presencialmente a partir de um questionário estruturado para coletar dados primários. A qualidade da informação financeira (QFI) foi avaliada pelo 'Escritório de Promoção de PMEs' a partir dos seguintes indicadores: nível de importância; satisfação; correlação satisfação x importância; habilidade com os negócios; e QIF, exatidão e completude, pontualidade e consistência. Os resultados mostraram que todos os indicadores foram significativos $(p<0,05)$ e com alta confiabilidade. Para a QIF o indicador mais válido foi exatidão e completude. A relação entre a QIF e a percepção da capacidade de acesso ao capital mostrou que a QFI diminuiu as ambiguidades quanto à posição financeira das PME e melhorou a confiança no acesso ao capital. A estimativa significativa de parâmetros apoia a relação entre QIF e desempenho financeiro, mostrando que aumenta a confiança do proprietário-gerente para buscar novas oportunidades, aumentando a performance da empresa. Os autores concluíram que a qualidade da informação financeira proporcionou acesso ao capital e aprimorou o desempenho das PME, permitindo que melhorassem sua disponibilidade de investimentos através da preparação e uso na tomada de decisões sobre requisitos financeiros. Bem como, que a qualidade da informação financeira tem significativo efeito no desempenho e na percepção dos proprietários-gerentes.

Shah, Shamail e Ahmad Akhtar (2015), no estudo Lean quality improvement model for quality practices in software industry in Pakistan, objetivaram mapear o ambiente e a cultura

Perspectivas em Gestão \& Conhecimento, João Pessoa, v. 9, n. 3, p. 64-80, set./dez. 2019. 
das pequenas e médias empresas de software (SMSH) no Paquistão para a melhoria da qualidade e de processos através da implementação de filosofia de gestão de qualidade. Para tanto, 48 questionários foram elaborados com o objetivo de descrever o nível de compreensão e implementação dos padrões de qualidade seguidos pelas SMSH. O questionário foi prétestado com uma pequena amostra de 30 respondentes e forneceu as informações iniciais que ajudaram a restringir o escopo da pesquisa. Um conjunto de oito construtos de qualidade (tamanho e estrutura da organização, cultura organizacional, comportamento organizacional, desenvolvimento de requisitos e gestão, planejamento de projetos, monitoramento e controle, medição e análise, e melhoria da qualidade do processo) foram mapeados como indicadores de desempenho para gerenciamento de qualidade e para a coleta de dados. Os autores propuseram, então, um framework para encontrar um conjunto mínimo de melhores práticas para a implementação do paradigma de gestão da qualidade na indústria local. Uma lista de 1031 empresas de software foi compilada pelo Pakistan Software Export Board e Pakistan Software House Association e aplicado em formato de questionário enviado por correio e por e-mail para preenchimento online. Assim, dos 725 questionários enviados pelo correio, $144(19,8 \%)$ retornaram e dos 90 aplicados por e-mail, 83 (92\%) foram respondidos. Do total de entrevistados, $7 \%$ estavam na alta gerência, incluindo o presidente, $39 \%$ na gerência intermediária, incluindo gerentes de programa e gerentes de linha, e 54\% na administração inferior, incluindo engenheiros de software, analistas de sistemas e pessoal de garantia de qualidade. A análise de correlação e de regressão de construtos de qualidade foi usada para determinar o nível das práticas de qualidade atuais, sendo que os sete constructos independentes foram significativos $(0,05)$ na análise de correlação e apenas quatro na análise de regressão. O comportamento organizacional foi inferior a 0,341, o teste Cronbach Alpha foi considerado significativo $(0,839)$ e o qui-quadrado ficou dentro da faixa aceitável $(2.32)$. Os autores concluíram que o modelo de melhoria de qualidade da informação forneceu direções inovadoras e flexíveis para as SMSH no Paquistão para mudar sua cultura de 'cultura corretiva' para 'preventiva' e para melhorar seus processos seguindo a filosofia da gestão da qualidade total (TQM).

Van Der Linden e Van De Leemput (2015) objetivaram apresentar a utilidade, a abordagem conceitual, a metodologia e os benefícios de um 'Observatório de Estudantes para uso de Ferramentas Baseadas em Computador' em uma universidade, no estudo intitulado Observatory of students uses of computer-based tools. Apresentaram a utilidade, a abordagem conceitual e os benefícios da criação do Observatory of Students' Uses of Computer-based Tools. Utilizaram uma metodologia de 4 passos (painel, focus groups, teste de usabilidade e questionário) e envolveram um sistema de gerenciamento de aprendizagem projetado como um portal informativo para estudantes e professores. Todos os estudantes universitários, no início de cada ano letivo, receberam um convite por e-mail para participar do painel e responder a um questionário. Destes 593 completaram o questionário, sendo 58\% mulheres. Este questionário visava identificar as características sociodemográficas dos estudantes, problemas tecnológicos encontrados na universidade e habilidade do aluno em participar de grupos focais ou de teste de usabilidade. Para avaliar a qualidade do sistema (QS) foram testadas a confiabilidade, flexibilidade, integração, acessibilidade e pontualidade do sistema; e para a qualidade da informação (QI), completude, precisão, formato e modo de apresentação das informações. Os resultados apontaram que $96,5 \%$ dos alunos tem habilidade com internet e 86,5\% autoeficácia com Tecnologia da Informação e Computação (TIC); 99,4\% usaram a internet para melhorar seu desempenho; a satisfação com o sistema foi 3,45 e com a informação 3,89; a usabilidade (0.507) e facilidade de uso (0.163) com impacto de $p<0,05$. Para QI os indicadores foram: relevância $(0,352)$, precisão $(0,384)$, completude $(0,137)$, compreensibilidade $(0,1223)$ e conteúdo $(0,105)$; e para QS: acessibilidade $(0,331)$, 
confiabilidade $(0,241)$, estética $(0,139)$, recursos $(0,138)$, flexibilidade $(0,123)$ e integração $(0,105)$. No painel a análise do conteúdo mostrou que os alunos se queixaram de confiabilidade, atualização de conteúdo, integração, layout, compatibilidade e problemas de utilidade entre tópicos. Ressaltaram que os grupos focais relataram problemas de ordem ergonômica e falta de utilidade, além de expressar o desejo de ter um portal mais personalizado. Para o teste de usabilidade, apesar da alta taxa de sucesso nas tarefas exigidas (10/12), os resultados mostraram uma variação intra e interrassunto importante no tempo necessário e no número de ações realizadas para completar a tarefa. Os autores observaram, a partir da análise pareada dos dois resultados, que os alunos estão menos satisfeitos com o sistema utilizado do que com a qualidade da informação apresentada. Concluíram que o Observatório possibilitou o intercâmbio entre autoridades e estudante, que o processo resultou em recomendações precisas e na definição de prioridades tem termos de ações a serem realizadas, e que a maioria dos alunos declararam boa capacidade para utilização de TIC, consolidando a ideia de que ações devem ser realizadas ao nível de sistema e gerenciamento aprendizagem.

Conforme observamos nos oito (8) estudos, a utilização da informação tem sido utilizada pelas organizações como ferramenta para antecipar tendências, para que estejam preparadas para o futuro e para que saibam desenvolver estratégias adequadas. Os observatórios, neste contexto, desempenham papéis relevantes, pois subsidiam as empresas nos processos de decisão e acabam por auxiliar na construção de uma visão de curto, médio e longo prazo.

\subsection{Risco de viés entre os estudos}

Com base no Best Practices for Survey Research Reports: A Synopsis for Authors and Reviewers da American Association for Public Opinion Research (AAPOR) (DRAUGALIS, 2008), encontramos viés nas seguintes questões e estudos:

- somente Shah; Shamail; Ahmad Akhtar (2015) reportaram usar designs que equilibram custos com erros (iii);

- os estudos de Bianchini et al. (2011), Carducci et al. (2011), Paschaloudis e Tsourela (2015) e Pérez e Nassif (2017) fizeram uso de instrumentos e não especificaram se houve a realização de pré-teste (v);

- não foi evidenciado em nenhum dos estudos o uso diferenciado para o tratamento de seres humanos (ix).

Para todas as demais questões de viés foi possível observar uma correlação nos estudos, bem como a sua aplicação.

\section{DISCUSSÃO DOS RESULTADOS}

Observamos que todos os estudos apontaram para um monitoramento contínuo da 'Qualidade da Informação' disponibilizada pelos 'Observatórios', ressaltando que estas interferem na tomada de decisão das organizações.

Apesar de o estudo de Sarapaivanich e Kotey (2006) e de Shah, Shamail e Ahmad Akhtar (2015) utilizarem o termo 'escritório' para posicionar este espaço de informações estratégicas sobre a qualidade da informação disponibilizada, estes adequam-se ao conceito proposto por Trzeciak (2009) para Observatório, motivo pelo qual foram considerados nesta

Perspectivas em Gestão \& Conhecimento, João Pessoa, v. 9, n. 3, p. 64-80, set./dez. 2019. 
pesquisa. Ambos estudos focaram em indicadores de qualidade e sua correlação com a percepção do cliente.

As informações que deram sustentação as análises de cada estudo vieram de entrevistas e questionários direcionados. Em alguns estudos a escala Likert proposta permitiu que os autores apresentassem uma análise quantitativa das informações colhidas. O que dificultou as análises foi a inexistência de metodologias específicas de avaliação da qualidade da informação.

Os estudos pontaram resultados advindos dos parâmetros pré-estabelecidos para a coleta das informações, não de instrumentos padronizados. O estudo de Paschaloudis e Tsourela (2015) foi o que apontou o uso de escalas ES-QUAL e E-RecS-QUAL para ajudar a identificar os pontos fortes e fracos nos sites e sugerir ideias para melhorias para a qualidade da informação. Percebe-se, porém, que o uso de instrumento de avaliação permite organizar o pensamento, já que aponta indicadores com percepções positivas e a melhorar, além da possibilidade de realizarmos correlações entre estudos para apontar destaques ou modelos a serem seguidos.

Quando correlacionamos os estudos de Carducci et al. (2011) com os de Clasen (1996), observamos que em ambos houve a necessidade de um monitoramento contínuo das informações de saúde disponibilizadas, ressaltando que seu impacto precisa ser estudado. A qualidade da informação foi fator preponderante para a tomada de decisão e pode ajudar a corrigir efeitos negativos que resultavam em informações erradas. Clasen (1996) não entrou na pesquisa principal porque seus estudos focaram em provedores isolados que acompanharam usuários dinamarqueses na internet, e não em um Observatório.

No tocante as Tecnologias de Informação e Comunicação (TIC), tanto os estudos de Sarapaivanich e Tokey (2006) quanto os de Van der Linden e Leemput (2015) mostraram que estas são críticas quando se avalia a qualidade da informação disponibilizada. Indicadores como nível de importância, satisfação, correlação entre satisfação e importância, habilidade com os negócios, exatidão e completude, pontualidade e consistência, ao serem usadas para medir a qualidade da informação demonstraram significativo efeito na percepção dos usuários (SARAPAIVANICH; TOKEY, 2006). Os indicadores da qualidade do sistema (confiabilidade, flexibilidade, integração, acessibilidade e pontualidade) e da qualidade da informação (completude, precisão, formato e informações apresentadas) também foram significativas para o gerenciamento da aprendizagem (VAN DER LINDEN; LEEMPUT, 2015). Os estudos seguiram o mesmo caminho, pois resultaram em recomendações mais precisas quanto a definição de prioridades em temos de ações que necessitam ser realizadas com o uso de tecnologia da informação e comunicação. Importante destacar que ambos os estudos apresentaram uma maior estruturação no sistema de medição da qualidade da informação, já que apontaram indicadores pré-definidos para avaliar as informações disponibilizadas.

Para finalizar, corroboramos com Calazans (2008) quando ressaltaram que a qualidade está baseada em sua própria percepção, e no atendimento a satisfação de suas necessidades e por não ser um conceito intangível existem muitas propostas de como medi-la e mensurá-la, utilizando medidas básicas fundamentais. Acreditamos ser este o principal desafio de todos os autores citados, visto que encontramos nos seus estudos a preocupação com a informação disponibilizada e a sua qualidade.

Perspectivas em Gestão \& Conhecimento, João Pessoa, v. 9, n. 3, p. 64-80, set./dez. 2019. 


\section{CONSIDERAÇÕES FINAIS}

É perceptível em todos os estudos realizados nos Observatórios que a qualidade da informação é um atributo importante e fornece insights para a tomada de decisão, permitindo entender melhor o contexto, as tendências, os desafios e os gaps.

Atendendo ao objetivo da pesquisa, ressaltamos que o foco na avaliação da qualidade da informação esteve presente em todos os estudos, porém encontramos dificuldades em fazer correlações entre os diferentes autores, já que não observamos similaridades nas amostras.

Medir a qualidade da informação, de acordo com os estudos, é essencial para a tomada de decisão, no entanto reforçamos que o uso de metodologias, métodos ou ferramentas estruturadas devem ser incorporadas nas estratégias dos Observatórios, já que direcionam as ações, delimitam gargalos e fornecem indicadores do que realmente precisa ser melhorado.

Indicadores como nível de importância, satisfação, correlação entre satisfação e importância, habilidade com os negócios, exatidão e completude, pontualidade e consistência, confiabilidade, flexibilidade, integração, acessibilidade e pontualidade, precisão e formato das informações formam um conjunto de indicadores significativos para o gerenciamento da qualidade da informação disponibilizada por Observatórios e foram apontados nos estudos, mesmo que de forma aleatória.

Em síntese, os estudos apontaram para uma preocupação com a qualidade das informações, pois estas estão relacionadas a tomada de decisão pelas organizações, podendo auxiliar em um redirecionamento de ações e, quando necessário, uma mudança de estratégia, conforme apontado pelos autores como um fator preponderante.

Ressaltamos que estudos que apresentem instrumentos de avaliação validados são necessários para mensurar a qualidade da informação, principalmente para aquelas disponibilizadas em Observatórios, já que estes atendem as demandas imediatas das organizações e influenciam na tomada de decisão.

\section{REFERÊNCIAS}

ALEXANDER, J. A.; HEARLD, L. R.; HASNAIN-WYNIA, R.; CHRISTIANSON, J. B.; MARTSOLF, G. R. Consumer trust in sources of physician quality information. Medical Care Research and Review, v. 68, n. 4, p. 421-440, 2011.

ALVES, J. B.; KERN, V. Teoria geral de sistemas. Florianópolis, 2007. Material do curso de TGA do Programa de Pós-Graduação em Engenharia e Gestão do Conhecimento. In: AMIN, E. H. F. Um modelo de gestão pública por indicadores de sustentabilidade em associação com observatórios urbanos. 2010. 203f. Tese (Tese de Doutorado). Programa de Pós Graduação em Engenharia e Gestão do Conhecimento. Universidade Federal de Santa Catarina, Florianópolis.

BACK, S. Modelo de Observatório para apoio ao processo de inovação nas organizações: aplicação para as indústrias brasileiras de Bens de Capital. 2016. 376 f. Tese (Doutorado em Ciência e Engenharia de Materiais) - Universidade Federal de Santa Catarina, Florianópolis.

BARBOSA, R. R. Gestão da informação e do conhecimento: origens, polêmicas e perspectivas. Informação \& Informação, Londrina, v. 13, n. esp., p. 1-25, 2008.

Perspectivas em Gestão \& Conhecimento, João Pessoa, v. 9, n. 3, p. 64-80, set./dez. 2019. 
BIANCHINI, A.; PELLEGRINI, M.; SACCANI, C. Material and energy recovery in integrated waste management system - An Italian case study on the quality of MSW data. Waste Management, v. 31, n. 9, p. 2066-2073, 2011.

BOOTH, P.; GASKELL, P.; HUGHES, C. The economics of data: quality, value \& exchange in web observatories. In: International Conference on World Wide Web, 22, 2013. Proceedings. ACM. p.1309-1316.

BOTHA, M.; BOTHA, A.; HERSELMAN, M. Data quality challenges: A content analysis in the ehealth domain. In: Information and Communication Technologies (WICT), 2014 Fourth World Congress. IEEE, 2014. p.107-112.

CALAZANS, A. T. S. Qualidade da informação: conceitos e aplicações. TransInformação, Campinas, v. 20, n. 1, p. 29-45, jan./abr. 2008.

CANTO, C. R. L. C.; VIOLADA, P. A. M. V.; TIBOCHA, C. Y. C.; FREIRE, P. de S.; SOUZA, J. A. Inovação disruptiva e a gestão do conhecimento: uma revisão sistemática. CONGRESSO BRASILEIRO DE GESTÃO DO CONHECIMENTO, KM BRASIL, 14., 2018. Anais [...]. 2018.

CANTO, C. R. L. C.; BASTOS, R. C.; BASTOS, L. Avaliação da qualidade da informação disponibilizada por Observatórios: revisão sistemática da literatura. ENANGRAD: Gestão da Aprendizagem, 30., 23 a 25 de agosto de 2019.

CARDUCCI, A.; ALFANI, S.; SASSI, M.; CININI, A.; CALAMUSA, A. Mass media health information: Quantitative and qualitative analysis of daily press coverage and its relation with public perceptions. Patient Education and Counseling, v. 82, n. 3, p. 475-478, 2011.

CARVALHO, E. L. Importância da gestão da informação para o processo decisório nas organizações. In: VALENTIM, M. L. P. (Org.). Informação, conhecimento e inteligência organizacional. 2. ed. Marília: FUNDEPE, cap. 5, p. 81-97. 2006.

CLAUSEN, H. Web information quality as seen from the libraries. New Library World, v. 97, n. 1130, p. 4-8, 1996.

DAVENPORT, Thomas H.; PRUSAK, Laurence. Conhecimento Empresarial: como as organizações gerenciam o seu capital intelectual. Rio de Janeiro: Campus, 1998.

DRAUGALIS, J. R.; COONS, S. J.; PLAZA, C. Best Practices for Survey Research Reports: A Synopsis for Authors and Reviewers. The American Journal of Pharmaceutical Education. v. 15; n. 72, p. 1-11, Feb, 2008.

FEDERAÇÃO DAS INDÚSTRIAS DO ESTADO DO PARANÁ (FIEP). Observatório Sesi/Senai/IEL. 2004. Disponível em: http://www.sistemafiep.org.br, acesso out/2017.

FERREIRA, A. B. de H. Novo dicionário da língua portuguesa. Rio de Janeiro: Nova Fronteira, 1975.

GARCIA-PEREZ-DE-LEMA, D.; MADRID-GUIJARRO, A.; MARTIN, D. P. Influence of universityfirm governance on SMEs innovation and performance levels. Technological Forecasting and Social Change, v. 123, n. Supplement C, p. 250-261, 2017.

Perspectivas em Gestão \& Conhecimento, João Pessoa, v. 9, n. 3, p. 64-80, set./dez. 2019. 
HAIQUN, M. Requirement for Information Quality in the Value-added Use of Public Sector Data. Information Studies: Theory \& Application, v. 10, p. 002, 2013.

HEY, J. The Data, Information, Knowledge, Wisdom Chain: the metaphorical link. dez. 2004. Disponível em: http://www.unesco.org/new/en/natural-sciences/ioc-oceans/. Acesso em: dia 11 jul. 2018.

LI, S.; LIN, B. Accessing information sharing and information quality assessment. Information \& Management, n. 40, p. 133-146, 2006.

MADNICK, S. E.; LEE, Y. W.; WANG, R. Y.; ZHU H. Overview and framework for data and information quality research. ResearchGate, June 2009.

MANOUSELIS, N.; COSTOPOULOU, C. Quality in metadata: a schema for e-commerce. Online Information Review, v. 30, n. 3, p. 217-237, 2006.

METTLER, T.; ROHNER, P.; BAACKE, L. Improving Data Quality in Health Information Systems: a holistic design-oriented approach. ResearchGate, jan. 2008.

MILLER, B.; MASEK, E.; MALLOY, M. A.; SELVAGEM, C. Towards a framework for managing the information environment. ResearchGate, v. 2, n. 4, p. 359-384, 2001.

MOHAMED, M.; MURRAY, A.; MOHAMED, M. The role of information and communication technology (ICT) in mobilization of sustainable development knowledge: a quantitative evaluation. Journal of Knowledge Management, v. 14, n. 5, p. 744-758, 2010.

PASCHALOUDIS, D.; TSOURELA, M. Using ES-QUAL to Measure Internet Service Quality of EBanking Web Sites in Greece. Journal of Internet Banking and Commerce, v. 19, n. 2, p. 1-17, 2015.

RADZIWILL, N. M. Foundations for quality management of scientific data products. Quality Management Journal, v. 13, n. 2, p. 7, 2006.

SANTOS, F. B.; TECCHIO, E.; FIALHO, F. A. P. Liderança autêntica e a gestão do conhecimento. Revista da Universidade Vale do Rio Verde, Três Corações, v. 12, n. 1, p. 579-588, jan./jul. 2014.

SARAPAIVANICH, N.; KOTEY, B. The effect of financial information quality on ability to access external funds and performance of SMEs in Thailand. Journal of Enterprising Culture, v. 14, $n$. 03, p. 219-239, 2006.

SHAH, F. T.; SHAMAIL, S.; AHMAD AKHTAR, N. Lean quality improvement model for quality practices in software industry in Pakistan. Journal of Software: Evolution and Process, v. 27, n. 4, p. 237-254, 2015.

STEIL, A. V.; PENHA, M. M.; BONILLA, M. A. M. Antecedentes da retenção de pessoas em organizações: uma revisão de literatura. Revista Psicologia Organização do Trabalho, v. 16, n. 1, jan-mar 2016.

VAN CANEGHEM, T.; VAN CAMPENHOUT, G. Quantity and quality of information and SME 
financial structure. Small Business Economics, v. 39, n. 2, p. 341-358, 2012.

VAN DER LINDEN, J.; VAN DE LEEMPUT, C. Observatory of studentsg uses of computer-based tools. Psychologie Française, v. 60, n. 2, p. 145-157, 2015.

VAN MOSSEVELD, C. et al. How to ensure quality of health accounts. Health Policy, v. 120, n. 5, p. 544-551, 2016.

VRÎNCIANU, M.; ANICA-POPA, L.; ANICA-POPA, I. Organizational memory: an approach from knowledge management and quality management of organizational learning perspectives. Amfiteatru Economic, v. 11, n. 26, p. 473-481, 2009.

YING, W. Research on Information Quality for Value-added Use of Information from Public Sectors [J]. Information Studies: Theory \& Application, v. 9, p. 012, 2012.

ZANG, T.; LIEBER, M.; NORTON, C.; FUCIK, K. The-advanced modeling, simulation and analysis capability roadmap vision for engineering. 2006. Conference Paper. 